\title{
Angiotensin-Converting Enzyme Inhibition: Beyond Blood Pressure Control-The Role of Zofenopril
}

\author{
Claudio Borghi $\cdot$ Stefano Omboni
}

Received: June 15, 2020 / Published online: August 8, 2020

(C) The Author(s) 2020

described. The focus of this review is centered on the role of ACEIs in addition to and beyond blood pressure control. It summarizes clinical evidence on the use of these agents in cardiovascular diseases, with a specific interest in the experience with zofenopril, which presents a peculiar pharmacological profile that may contribute to additional clinical benefits in some identifiable populations of patients. Indeed, the presence of a sulfhydryl group in its structure confers on zofenopril high anti-oxidant and anti-ischemic properties involving the activation of the $\mathrm{H}_{2} \mathrm{~S}$ system, resulting in a cardioprotective effect. The efficacy and safety of zofenopril have been extensively evaluated and proved in the Survival of Myocardial Infarction Long-Term Evaluation (SMILE) program in numerous clinical settings. The pharmacological features and ancillary characteristics of zofenopril with potent cardioprotective effects seem to differentiate it from other ACEIs and to confer further benefits to patients.

C. Borghi $(\bowtie)$

Unit of Internal Medicine, Policlinico S. Orsola,

University of Bologna, Bologna, Italy

e-mail: claudio.borghi@unibo.it

S. Omboni

Clinical Research Unit, Italian Institute of

Telemedicine, Varese, Italy

S. Omboni

Scientific Research Department of Cardiology,

Science and Technology Park for Biomedicine,

Sechenov First Moscow State Medical University,

Moscow, Russian Federation

Keywords: ACE inhibitor; Bradykinin; Myocardial infarction; SMILE study; Sulfhydryl group; Zofenopril 
Key Summary Points

Angiotensin-converting enzyme inhibitors (ACEIs) are currently recommended for managing arterial hypertension, the progression of chronic renal disease, post-myocardial infarction, congestive heart failure, and type 2 diabetes mellitus.

In patients with acute or chronic coronary syndrome and heart failure ACEIs appear to provide several benefits beyond blood pressure control.

Among the various drugs of this class, zofenopril, a lipophilic, sulfhydryl groupcontaining ACEI, seems to contribute to additional clinical benefits in some identifiable populations of subjects.

The clinical efficacy and safety of zofenopril has been evaluated in more than 3,600 post-acute myocardial infarction patients with or without left ventricular dysfunction in the four randomized controlled trials of the SMILE program.

The pharmacological characteristics and ancillary features of zofenopril with potent cardioprotective activity seem to differentiate it from other ACEIs and to confer further benefits to patients.

\section{INTRODUCTION}

Given the consolidated clinical experience in the use of therapies blocking the renin-angiotensin system (RAS), angiotensin-converting enzyme inhibitors (ACEIs) and angiotensin IIreceptor blockers (ARBs) have become a firstline strategy for managing arterial hypertension, the progression of chronic renal disease, post-myocardial infarction, congestive heart failure, and type 2 diabetes mellitus [1,2]. These results have been generated by a large number of randomized clinical trials and observational post-surveillance studies that have demonstrated the clinical efficacy of RAS blockade in daily practice and in patients with presumed RAS activation and without hypertension [2]. The focus of this review is centered on the role of ACEIs beyond blood pressure (BP) control and summarizes the clinical evidence on the use of these agents in patients with different settings of cardiovascular diseases (CVDs). Specific attention is paid to the experience with zofenopril because of its peculiar pharmacological properties, mainly related to the sulfhydryl group present in its molecular structure. This article is based on previously conducted studies and does not contain any studies with human participants or animals performed by any of the authors.

\section{RAS INVOLVEMENT IN CVD}

The RAS is one of the main physiological system responsible for homeostatic control of arterial pressure, electrolyte balance, organ perfusion, and extracellular volume. Its modulation has several important effects that mainly involve CVDs and renal disease [3]. The pathway is initiated by renin which cleaves the N-terminal portion of angiotensinogen to form the biologically inactive decapeptide angiotensin I [4]; this inactive peptide is then hydrolyzed by ACE which removes the C-terminal dipeptide in order to form the octapeptide angiotensin II, biologically active as a vasoconstrictor [4]. Angiotensin II is the primary effector of a variety of RAS-induced physiological and pathophysiological actions. It interacts with 4 receptor subtypes expressed in the $\mathrm{CV}$ system (vasoconstriction, increased BP, increased cardiac contractility, vascular and cardiac hypertrophy), kidney (renal tubular sodium reabsorption, inhibition of renin release), sympathetic nervous system, and adrenal cortex (stimulation of aldosterone synthesis), as well as in the lung and the gut where it plays a remarkable role in fluid balance and respiratory function $[5,6]$.

Therefore, a dysregulation of RAS is reported not only in essential hypertension and in the 
response of $\mathrm{CV}$ and renal tissue to hypertensive and non-hypertensive injury but also in many other CV disorders [4]. Angiotensin II is involved in the development of both vascular and cardiac hypertrophy and remodeling, vascular damage, renal damage, and atherosclerosis with a mechanism that is only partially dependent on elevated BP values. [4]. In congestive heart failure (HF), the reduced cardiac function and the renal under-perfusion related to a reduced effective intravascular volume are responsible for renin hypersecretion and secondary aldosteronism, contributing to pulmonary congestion and development of edema. In these patients, angiotensin II increases peripheral vascular resistance (cardiac afterload) and enhances progressive ventricular

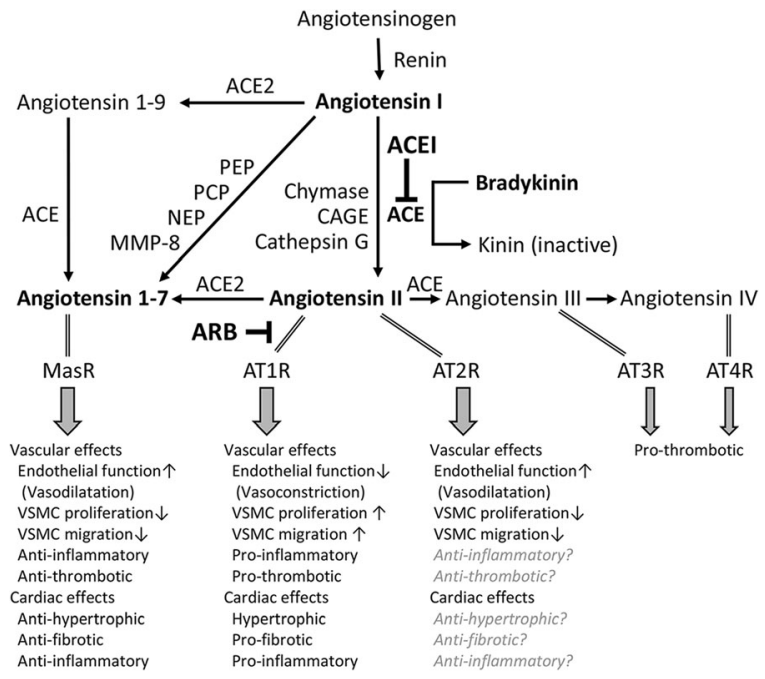

Fig. 1 Renin-angiotensin system and related molecules. Many molecules influence the effects of renin-angiotensin system activation. Angiotensin II and AT1R are key molecules of the major harmful pathway in the cardiovascular system. Counteracting pathways with cardiovascular protective property also exist. There are controversies about the effects of AT2R signaling, which are indicated in gray italic [3]. ARB angiotensin receptor blocker, AT1R angiotensin type one receptor, $A T 2 R$ angiotensin type two receptor, $A T 3 R$ angiotensin type three receptor, $A T 4 R$ angiotensin type four receptor, $C A G E$ chymostatin-sensitive angiotensin-II-generating enzyme, Mas $R$ Mas receptor, $M M P-8$ matrix metalloproteinase-8, NEP neutral endopeptidase, PCP prolyl carboxypeptidase, PEP prolyl endopeptidase, $R A S$ renin-angiotensin system, VSMC vascular smooth muscle cell dysfunction contributing to the progression of heart failure [4].

ACEI and ARB medications both target RAS, and are recommended in the treatment of several types of CVD (Fig. 1). However, the optimal choice between these two groups of medications for the prevention of CVD is not well established [3].

ACEIs prevent the conversion of angiotensin I to angiotensin II by competing with angiotensin I for the active site of ACE. The reduction of angiotensin II formation decreases vasoconstriction and aldosterone secretion and increases plasma renin. As a consequence, ACEIs can lower BP, total peripheral resistance, and sodium and water retention [7]. ACEIs also reduce both preload and afterload through arterial and venous dilation. These hemodynamic effects are particularly beneficial in the presence of left ventricular dysfunction (LVD) [8]. One disadvantage of ACEIs is that the presence of non-ACE pathways results in continued low-level production of angiotensin II, despite the inhibition of ACE. ARBs are selective ligands of AT1 receptors; these drugs achieve their clinical goals bypassing the limitations of ACE escape phenomena and non-ACE sources of angiotensin II formation, despite the increased blood angiotensin II levels due to blockade of AT1-mediated receptor internalization (Fig. 1) [3, 9].

\section{ROLE OF ACEIS IN CVD ACCORDING TO CURRENT GUIDELINES}

ACEIs are widely prescribed to manage hypertension, and are recommended in recent guidelines (level of recommendation IA) as the basis of antihypertensive strategies, given their ability to reduce $\mathrm{BP}$ and $\mathrm{CV}$ events in randomized clinical trials [10]. The potency of ACE inhibition is influenced by the affinity of the drug to the zinc $(\mathrm{Zn}++)$ ligand of ACE. There are three distinct chemical classes of ACEIs (Table 1): (1) sulfhydryl containing ACEIs (i.e., captopril, zofenopril) which strongly bind the $\mathrm{Zn}++$ ligand, but disulfide formation limits their half-life; (2) drugs containing a carboxyl 


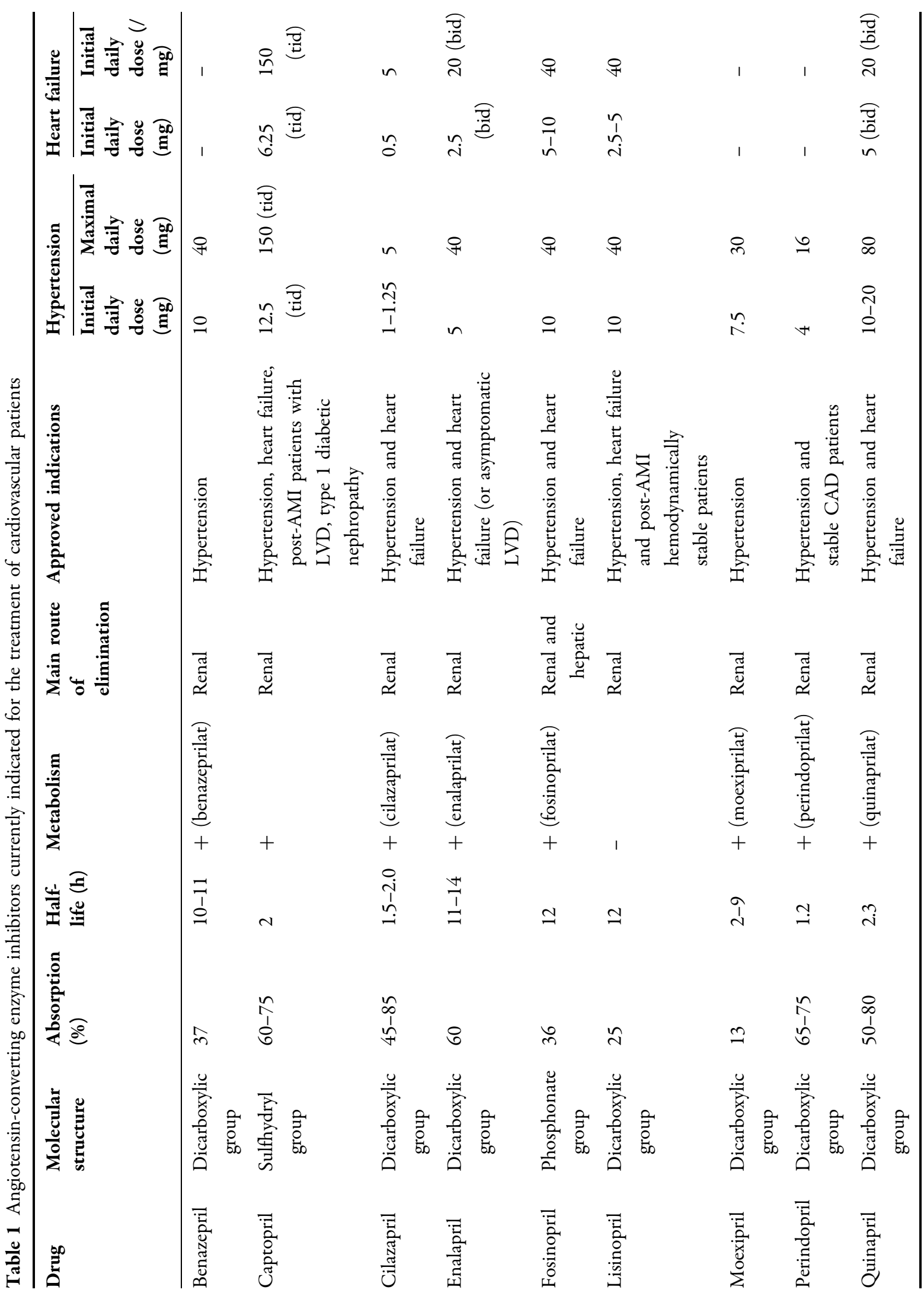




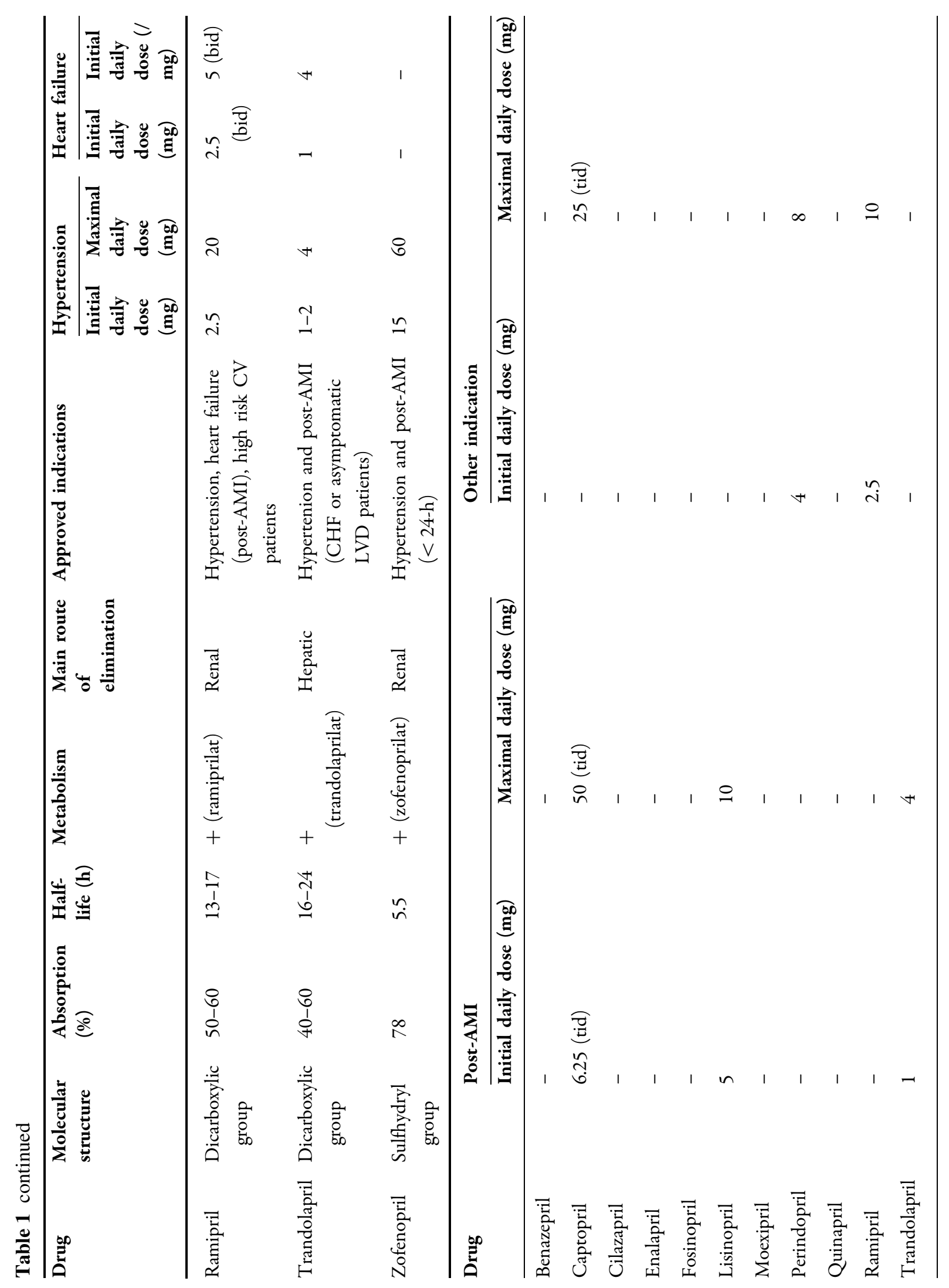


group (i.e., enalapril, lisinopril, ramipril) that binds to side chains of the enzyme within the active moiety for improved potency and duration of action; and (3) drugs composed of phosphorus-containing ACEIs (i.e., fosinopril) $[11,12]$. Although direct ACEIs comparisons among available agents are rare, a Cochrane review has demonstrated that there are no clinically meaningful differences in BP-lowering efficacy between different ACEIs [13].

ACEIs are the mainstay not only for hypertension management but also for other CVD, including HF, LVD, and acute myocardial infarction (AMI) (Table 2). In HF, ACEIs decrease total peripheral resistance, pulmonary vascular resistance, pulmonary capillary wedge pressure, and mean arterial and right atrial pressures, thus resulting in reduced mortality, regardless of HF severity [7, 8]. In these patients, the use of ACEIs has been shown to increase cardiac index, cardiac output, stroke volume, and exercise tolerance [7]. Many pieces of evidence indicate that BP control delays the onset of $\mathrm{HF}$ and, in some cases, contributes to prolonging life, and this also applies to hypertensive patients treated with ACEIs with or without a history of myocardial infarction, especially in older people [14]. As per current guidelines, an ACEI is recommended in addition to a betablocker for symptomatic patients with HF with reduced left ventricular ejection fraction (LVEF) to reduce the risk of HF hospitalization and death (recommendation 1A) [15]. A meta-analysis of three randomized trials (SAVE, Survival and Ventricular Enlargement; AIRE, Acute Infarction Ramipril Efficacy; and TRACE, trandolapril in patients with reduced LVD after acute myocardial infarction), including 5966 patients with LVD or HF, have indicated that administration of ACEIs after AMI provides clear benefit and lead to substantial reductions in mortality and morbidity in high-risk patients [16]. Indeed, the proportion of patients readmitted for HF resulted was reduced by $27 \%$ with ACEIs $[11.9 \%$ in the ACEI group vs. $15.5 \%$ of the control group; hazard ratio (HR) $0.7395 \%$ CI $0.63-0.85, p<0.0001]$, while the combination of death or hospital admission for HF occurred in $30.5 \%$ of the ACEI group and $37.0 \%$ of the control group [17]. ACEIs are also 
Table 2 Current recommendations of the European Society of Cardiology for the use of angiotensin converting enzyme inhibitors in patient with cardiovascular disease

\begin{tabular}{lll}
\hline & $\begin{array}{l}\text { Class of } \\
\text { recommendation }\end{array}$ & $\begin{array}{l}\text { Level of } \\
\text { evidence }\end{array}$ \\
\hline
\end{tabular}

Recommendations for treating arterial hypertension [10]

Among all antihypertensive drugs, ACEIs, ARBs, BBs, CCBs, and diuretics (thiazides I

A and thiazide-like drugs such as chlorthalidone and indapamide) have demonstrated effective reduction of $\mathrm{BP}$ and $\mathrm{CV}$ events in RCTs, and are thus indicated as the basis of antihypertensive treatment strategies

Combination treatment is recommended for most hypertensive patients as initial I

A therapy. Preferred combinations should comprise a RAS blocker (either an ACEI or an $\mathrm{ARB}$ ) with a $\mathrm{CCB}$ or diuretic. Other combinations of the five major classes can be used

It is recommended that if $\mathrm{BP}$ is not controlled with a two-drug combination, I

A treatment should be increased to a three-drug combination, usually a RAS blocker with a CCB and a thiazide/thiazide-like diuretic, preferably as an SPC

Recommendations for treating patients with symptomatic HF with reduced ejection fraction [15]

An ACEI is recommended, in addition to a BB, for symptomatic patients with HFrEF I

A to reduce the risk of HF hospitalization and death

$\mathrm{A} B \mathrm{~B}$ is recommended, in addition to an ACEI, for patients with stable, symptomatic I HFrEF to reduce the risk of HF hospitalization and death

An MRA is recommended for patients with HFrEF who remain symptomatic despite I treatment with an ACEI and a BB to reduce the risk of HF hospitalization and death

Recommendations for treating chronic coronary syndrome [18]

ACEIs (or ARBs) are recommended if a patient has other conditions (e.g., HF, hypertension, or diabetes)

ACEIs should be considered in CCS patients at very high risk of CV events

IIa

A

Recommendations for treating STEMI [22]

ACEIs are recommended, starting within the first 24-h of STEMI in patients with I evidence of HF, LVSD, diabetes, or anterior AMI

An ARB, preferably valsartan, is an alternative to ACEIs in patients with HF and/or I

A

A

LVSD, particularly those who are intolerant to ACEIs

ACEIs should be considered in all patients in the absence of contraindications

IIa

A

$A C E I$ angiotensin-converting enzyme inhibitor, $A R B$ angiotensin receptor blocker, $B P$ blood pressure, $B B$ beta-blocker, $C C B$ calcium channel blocker, $C C S$ chronic coronary syndrome, $C V$ cardiovascular, $H F$ heart failure, $H F r E F$ heart failure with reduced ejection fraction, $L V S D$ left ventricular systolic dysfunction, $M R A$ mineralocorticoid receptor antagonist, $R A S$ renin-angiotensin system, $R C T$ randomized controlled trial, SPC single-pill combination, STEMI ST-segment elevation myocardial infarction 
indicated in patients with asymptomatic LV systolic dysfunction in order to minimize the risk of development of $\mathrm{HF}$, and consequent hospitalization, and death [15].

In patients affected by coronary artery disease (CAD) with preserved systolic ventricular function, the evidence for routine administration has been conflicting. A metanalysis by AlMallah et al. reported a modest benefit of ACEIs on the prognosis of patients with CAD associated with preserved LV systolic function [17]. Data from more than 33,000 patients indicated that ACEIs therapy, when added to conventional therapy (aspirin, beta-blockers, and statins), was associated with a decrease in the rate of CV mortality (risk reduction or RR $0.83,95 \%$ CI 0.72-0.96, $p=0.01$ ), nonfatal MI (RR 0.84, 95\% CI 0.75-0.94, $p=0.003)$, all-cause mortality (RR $0.87,95 \%$ CI $0.81-0.94, p=0.0003$ ), and coronary revascularization (RR $0.93,95 \%$ CI $0.87-1.00, p=0.04$ ) [17]. Not all trials, however, described an effective advantage in the use of ACEIs in this setting; hence, current guidelines generally do not recommend ACEIs in chronic coronary syndrome patients without HF or high $\mathrm{CV}$ risk, unless with coexisting hypertension, LVEF $\leq 40 \%$, diabetes, or chronic kidney disease. In these latter cases, ACEIs should be considered (level of recommendation IIA) [18].

Therapy with ACEIs is also common after AMI. Numerous trials have indicated that mortality and morbidity were reduced when ACEIs were administered in a relatively unselected population of patients in the acute phase of MI ("early") or in patients with evidence of LVD in the subacute phase after MI ("late") [19]. In their metanalysis, Franzosi et al. collected data from large randomized trials which evaluated the efficacy and safety of treatments with ACEIs given in the acute phase of MI (0-36 $\mathrm{h}$ from the onset of symptoms) and continued for a short period of follow-up (generally 4-6 weeks) [20]. These trials were CONSENSUS-II, GISSI-3, ISIS-3, and CCS-1, and included a total of 98,496 patients. The main conclusions of the analysis were: (1) ACEI treatment can be started immediately during the acute phase of MI, in association with other routinely recommended treatments (such as thrombolytics, aspirin, and beta-blockers); (2) the benefit occurs during the first few days after MI, suggesting a positive role of tissue remodeling; (3) the benefit is proportionally larger in higher-risk subgroups (those with an anterior site MI or high heart rate); and (4) early benefits would be complementary to that observed later in trials of prolonged ACEI therapy initiated several days or weeks after MI in patients with evidence of HF or LVD [20]. Due to the lack of clinical trials that directly compared the efficacy of different ACEIs treatment after AMI, these drugs are generally assumed to be equally effective [21]. Analyzing the association between the choice of an ACEI after MI with the risk for mortality and reinfarction, a class effect was described rather than a superiority of some agents, when drugs were used in comparable dosages, thus suggesting that it would be more important initiating treatment and continuing treatment at the recommended dosage than choosing a particular agent to achieve long-term benefits [21]. Current guidelines recommend ACEIs after AMI with ST-elevation, starting within the first $24 \mathrm{~h}$ of ST-elevation myocardial infarction (STEMI) in patients with evidence of heart failure, LV systolic dysfunction, diabetes, or an anterior infarct (level of recommendation IA). They should be considered in all patients with AMI unless specifically contraindicated (level of recommendation IIa) [22].

ACEIs are commonly used in patients with diabetes and/or with renal disease. In a systematic review including 36,917 participants (including 2400 deceased, 766 patients who required dialysis, and 1099 patients in which serum creatinine levels doubled), ACEIs showed higher probabilities of reducing all-cause mortality, use of dialysis, or doubling of serum creatinine levels, and demonstrated renoprotective effects [23]. These observations were confirmed even in chronic renal disease progression when ACEIs decreased $\mathrm{BP}$, and urinary protein excretion slowed the increase in serum creatinine and reduced the risk for end-stage renal disease (ESRD), or for the combined outcome of doubling of the baseline serum creatinine concentration or ESRD by approximately 30\% [24]. 
Table 3 Pharmacological properties of sulphydrylic angiotensin-converting enzyme inhibitors[25]

\begin{tabular}{|c|c|}
\hline Pharmacological properties & Clinical effects \\
\hline Higher lipophilicity and tissue penetration & Effective prevention of $\mathrm{CV}$ events \\
\hline $\begin{array}{l}\text { Higher affinity and more persistent binding to tissue ACE } \\
\text { (cardiac, renal and vascular) }\end{array}$ & Effective prevention of $\mathrm{CV}$ events \\
\hline Lesser Bk-dependent effect & $\begin{array}{l}\text { Good safety (reduced frequency of hypotension, cough, and } \\
\text { renal function deterioration) }\end{array}$ \\
\hline Significant antioxidant effect & Significant anti-ischemic effect \\
\hline Increased release and availability of $\mathrm{H} 2 \mathrm{~S}$ & Improved CV function \\
\hline
\end{tabular}

$A C E$ angiotensin-converting enzyme, $B K$ bradikinin, $C V$ cardiovascular

\section{ZOFENOPRIL: A CARDIOPROTECTIVE ACEI}

Zofenopril is a sulfhydryl ACEI featuring a high lipophilicity, long-lasting tissue penetration, selective cardiac ACEI, potent anti-oxidant activities, and good efficacy after a single daily administration (Table 3) [25]. Starting from 1990, its efficacy and safety have been evaluated in the Survival of Myocardial Infarction LongTerm Evaluation (SMILE) project, a large clinical program aimed at investigating the role of zofenopril in the treatment of AMI in several populations of patients to test the hypothesis that its unique pharmacological profile could provide some benefits beyond ACE inhibition, in terms of $\mathrm{CV}$ event prevention (Table 4). The first trial was a pilot study that evaluated the safety of zofenopril administration within $24 \mathrm{~h}$ of the onset of AMI symptoms and provided some preliminary data of efficacy in terms of combined occurrence of death, non-fatal CV events, and severe adverse events after a 12-month follow-up [26]. A large clinical trial was then designed to determine the efficacy of 6-week zofenopril administration (15-60 mg twice daily) versus placebo in 1556 patients with MI not receiving thrombolysis and treated within $24 \mathrm{~h}$ of the onset of symptoms (SMILE$1)$. The incidence of death or severe congestive HF at 6 weeks (primary end-point) was significantly reduced in the zofenopril group, with risk reduction by $34 \% \quad(95 \%$ CI $8-54 \%$; $p=0.018)$. These positive outcomes were maintained after 1 year, observing a risk reduction for death of $29 \% \quad(95 \%$ CI $6-51 \%$; $p=0.011$ ) [27]. Given the large size of population involved in the SMILE trials, many statistically pre-specified and adequately powered post hoc analyses on specific subgroups could be performed, providing evidence on zofenopril effects in older patients, and in patients with arterial hypertension [28], diabetes [29], hypercholesterolemia [30], metabolic syndrome [31], angina, or previous myocardial infarction or NSTEMI [32]. Zofenopril was then directly compared with two ACEIs in randomized clinical trials carried out in patients with coronary artery disease. In the SMILE-2 study, zofenopril was compared to lisinopril in 1024 patients with acute MI undergoing thrombolysis [33], while in the SMILE-4 study, the main comparison was between zofenopril and ramipril in combination with acetylsalicylic acid (ASA) in patients with post-MI left ventricular dysfunction. In the SMILE 2 trial, zofenopril slightly reduced the incidence of severe hypotension versus lisinopril $(10.9 \%$ vs. $11.7 \%, p=0.38)$, with a significant decrease in the rate of drug-related severe hypotension $(6.7 \%$ vs. $9.8 \%, p=0.048)$ without a difference in mortality or major CV complications [33]. SMILE-4 investigated whether the benefits of zofenopril or ramipril could be affected by a concomitant ASA administration in patients with LV dysfunction after AMI [34]. The occurrence of death or hospitalization for $\mathrm{CV}$ cause was significantly reduced with zofenopril (odds ratio: 0.70; 95\% CI: 0.51-0.96; 


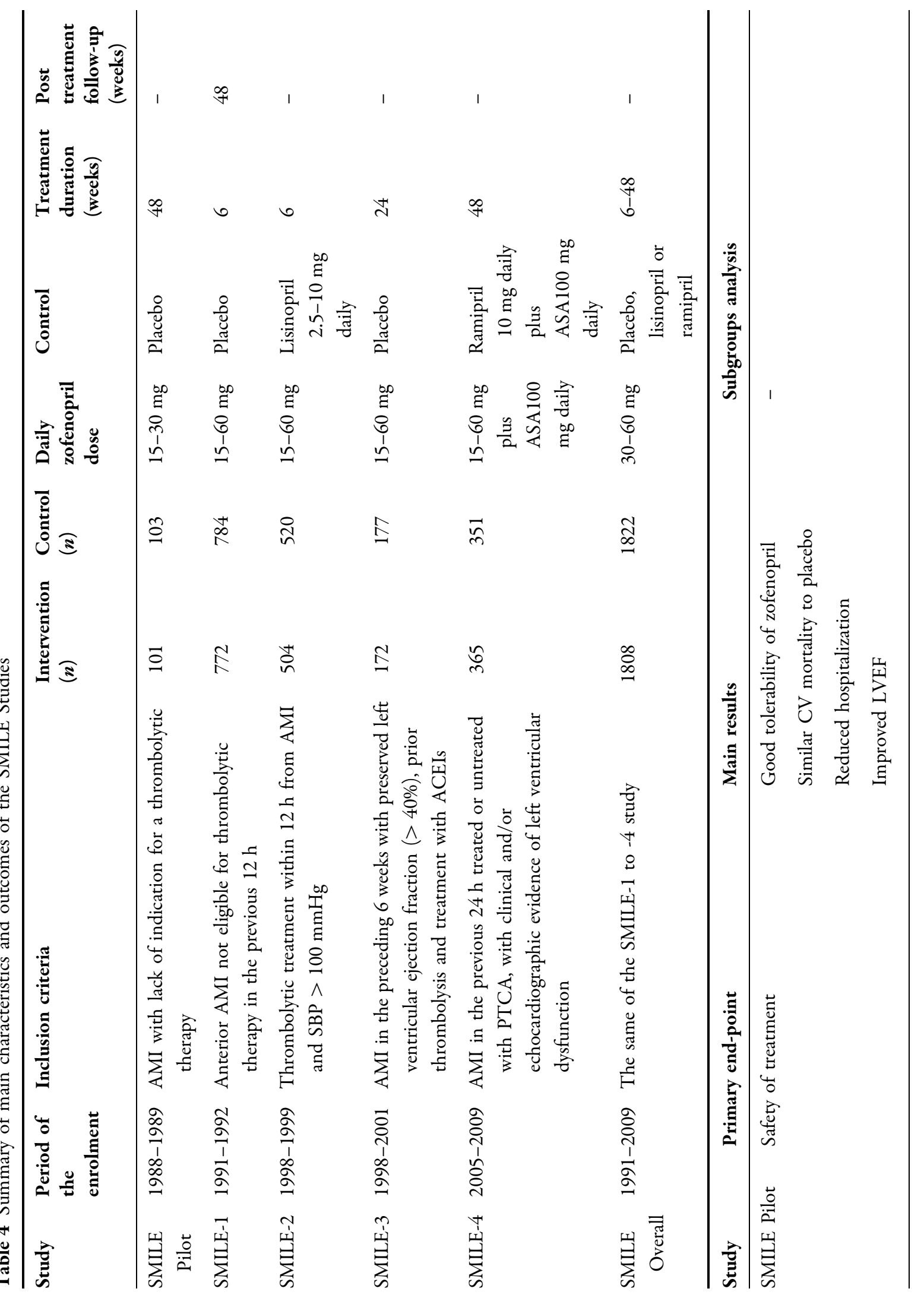




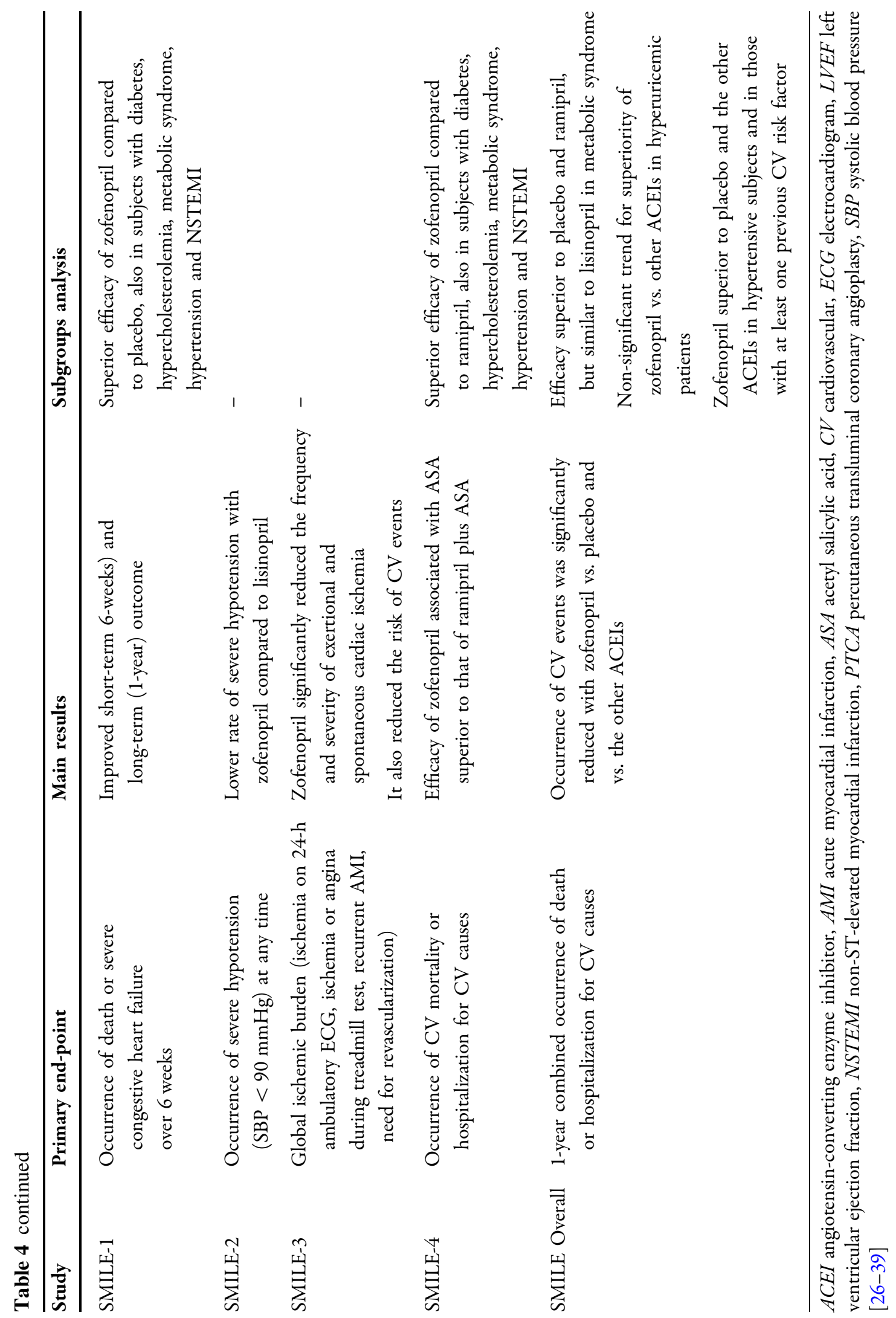


$p=0.028)$, and this benefit was maintained during 5-year follow-up (Fig. 2) [34, 35]. The same results have been confirmed after a propensity analysis of the population and comparing subpopulations of patients appropriately matched for the main prognostic variables [36]. Finally, the cardioprotective effects of zofenopril were investigated in the SMILE-3 ISCHEMIA trial in 349 patients with preserved LV function after MI. In these patients receiving recommended standard treatment, zofenopril reduced the global burden of myocardial ischemia by decreasing the occurrence of significant ST-T abnormalities on ambulatory electrocardiography (ECG), ECG abnormalities or symptoms of angina during the standard exercise test, MI recurrence and the need for revascularization procedures, in comparison to placebo (20.3\% vs. $35.9 \%, p=0.01$ ) (Fig. 3) [37]. The results were not affected by demography, BP control, and concomitant pharmacological treatment, and supported some primary cardioprotective effect of zofenopril. More recently, the results of the SMILE-ischemia study were corroborated by a post hoc analysis of SMILE 4, stratifying patients by LVEF measured at baseline, and showing a reduction in 1 year and long-term mortality and hospitalization in post-MI with preserved LV function (Fig. 4) [38]. The cardioprotective properties of zofenopril were confirmed in a pooled analysis of the four SMILE studies [39] (Fig. 5).

\section{THE RATIONALE OF THE CARDIOPROTECTIVE PROPERTIES OF ZOFENOPRIL}

Zofenopril-mediated cardioprotection during ischemia/reperfusion seems to be associated with an increased $\mathrm{H}_{2} \mathrm{~S}$ and nitric oxide (NO) signaling. Hydrogen sulfide is a cytoprotective physiological signaling molecule acting in concert with $\mathrm{NO}$ and carbon monoxide in order to maintain physiological homeostasis in both the heart and circulation. Experimental evidence indicates that $\mathrm{H}_{2} \mathrm{~S}$ donors, such as zofenopril, can attenuate the pathological consequences of myocardial ischemia/reperfusion injury and $\mathrm{HF}$, reducing oxidative stress and inflammation and inhibiting apoptosis [40]. In experimental models, indeed, zofenopril preconditioning enhanced tissue antioxidant defense, thus preventing reactive oxygen species formation, following an ischemic injury during reperfusion. Furthermore, treatment with zofenopril upregulated the expression of antioxidant enzymes, such as Trx-1, GPx-1, and SOD-1, suggesting an enhancement in antioxidant defenses before ischemia that mitigate myocardial reperfusion injury [41]. As summarized in Fig. 6 , the enhanced $\mathrm{H}_{2} \mathrm{~S}$ signaling, alongside the inhibition of angiotensin II formation and bradykinin metabolism, could represent a further explanation of additional beneficial effects provided by zofenopril [40]. In addition, ex vivo animal studies indicated that, at the heart level, where ACE inhibition may prevent ischemic damage, zofenopril produced a more striking and long-lasting ACE inhibition than ramipril and enalapril, and, to a lesser extent, than captopril and fosinopril; $60 \%$ of ACE inhibition could persist in the heart at least $8 \mathrm{~h}$ after administration, while it was much lower with other ACEIs [41]. Conversely, in the lung, zofenopril showed a lower potency in both inhibiting ACE and reducing bradykinin metabolism [41]; bradykinin accumulation in the airways with zofenopril is lower than that observed with other ACEIs, and this may partially explain the relatively low risk of coughing observed with zofenopril (Fig. 7) [42]. Indeed, a meta-analysis including hypertensive and postmyocardial infarction patients indicated that zofenopril-induced cough was generally of a mild to moderate intensity, occurred significantly $(p=0.001)$ more frequently in the first $3-6$ months of treatment $(3.0 \%$ vs. $0.2 \%$ 9-12 months), and always resolved or improved upon therapy discontinuation [39].

\section{CONCLUSIONS}

The pharmacological features of zofenopril provide cardioprotective, anti-ischemic, and anti-oxidant effects that differentiate it from other ACEIs. Zofenopril was evaluated as an early treatment after AMI, showing both shortand long-term benefits, and it has been 

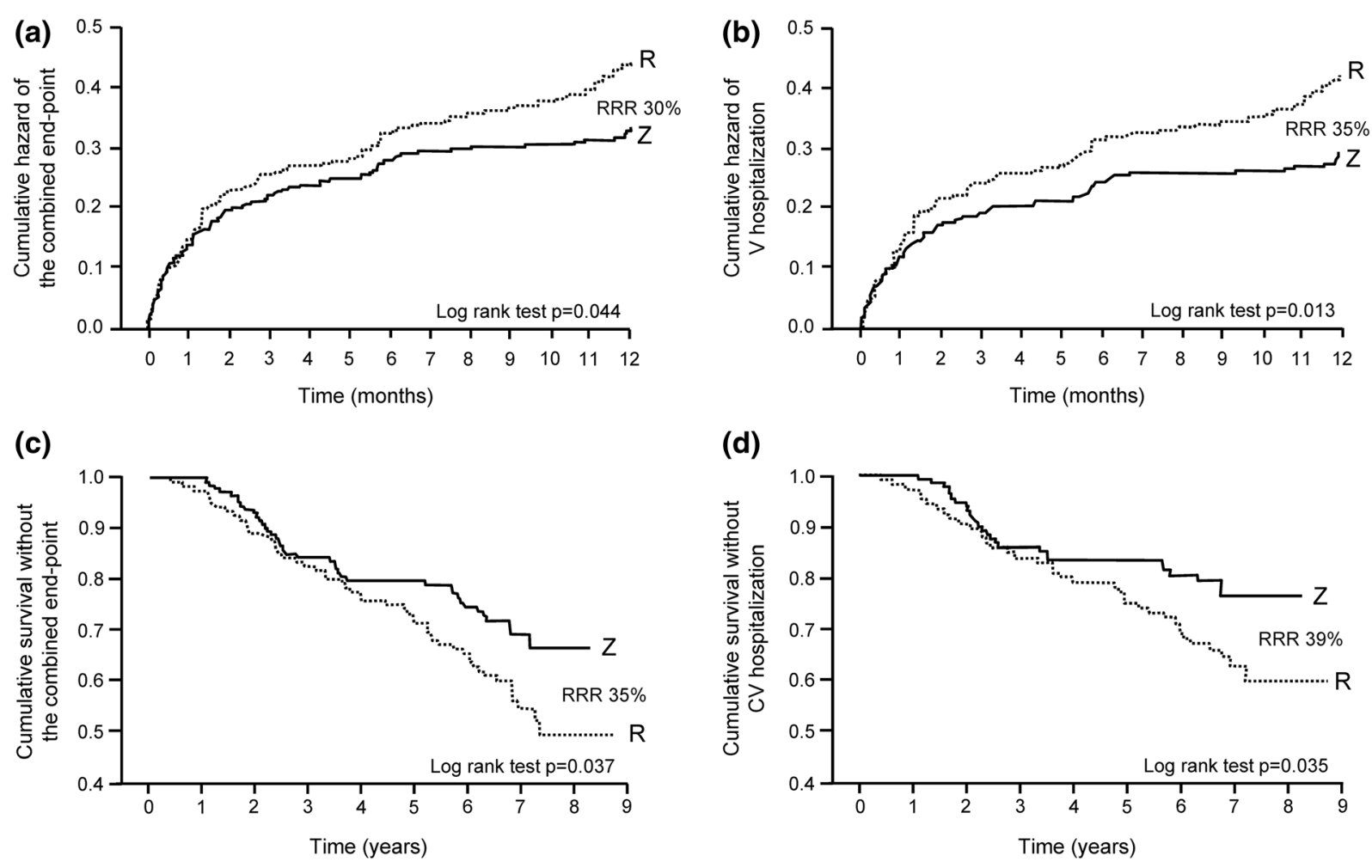

Fig. 2 a Incidence of the combined end-point (cardiovascular mortality or hospitalization for cardiovascular causes) and $\mathbf{b}$ of hospitalization during the 1-year of treatment with ramipril plus acetylsalicylic acid $(n=351$, dashed lines) or zofenopril plus acetylsalicylic acid $(n=365$, continuous lines) in the SMILE-4 Study. $\mathbf{c}$ Incidence of the combined endpoint and $\mathbf{d}$ hospitalization during the

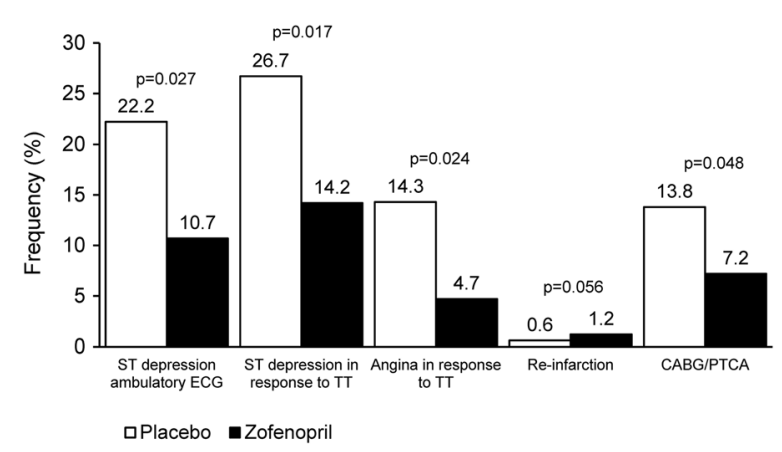

Fig. 3 Occurrence (\%) of the different primary endpoints of the study after 6 months of treatment with placebo ( $n=177$, open bars $)$ or zofenopril $(n=172$, full bars $)$ in the patients of the SMILE-3 Study [37]. TT treadmill test, $C A B G$ coronary artery bypass graft, PTCA percutaneous transluminal coronary angioplasty. $p$ values refer to the statistical significance of the between-treatment difference

follow-up in patients originally randomized and treated with zofenopril (continuous lines, $n=144$ ) or ramipril (dashed lines, $n=121$ ) in the SMILE-4 Study. $p$ values from the log-rank statistics $[34,35] . R R R$ relative risk reduction, $R$ ramipril, $Z$ zofenopril

confirmed as a feasible and effective therapy in many subgroups of patients with $\mathrm{CV}$ and metabolic comorbidities. Therefore, as well as other ACEIs, zofenopril is recommended for the management of CVD, including HF, AMI, and LVD, beyond BP control. As per current guidelines, ACEIs are recommended in symptomatic patients with HF with reduced LVEF to reduce the risk of HF hospitalization and in patients after AMI with ST-elevation. Since the effect in reducing the risk of mortality and reinfarction is not specifically associated with a single agent, and is rather described as a class effect, the choice of ACEI therapy can be addressed by the ancillary characteristics of each agent that may confer further benefits to patients. 

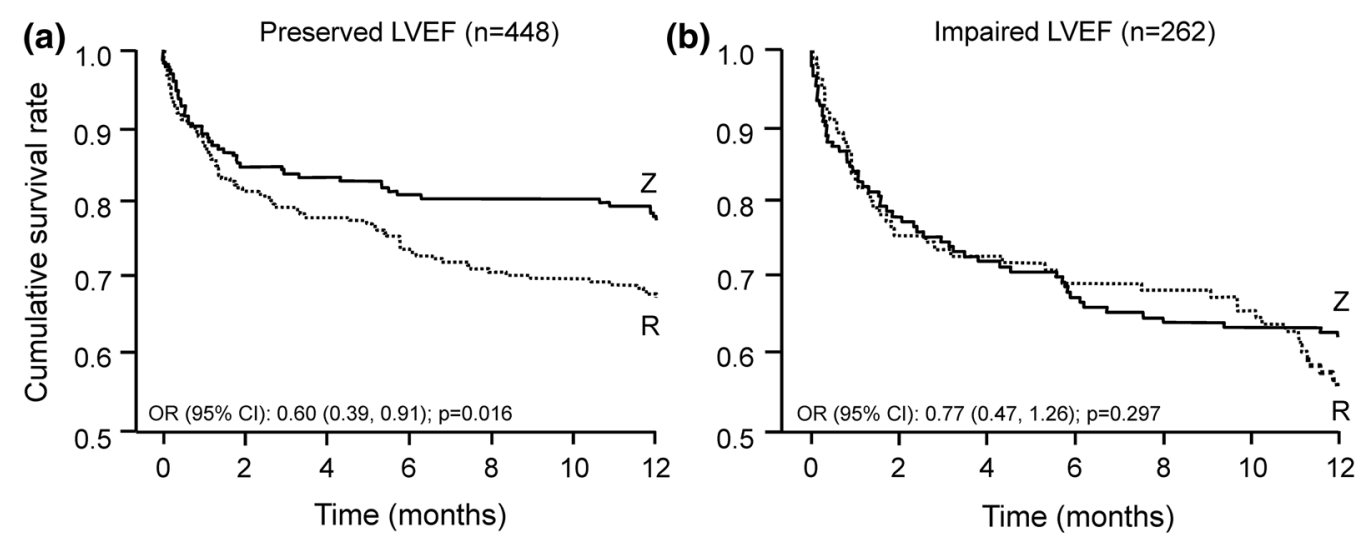

(c)

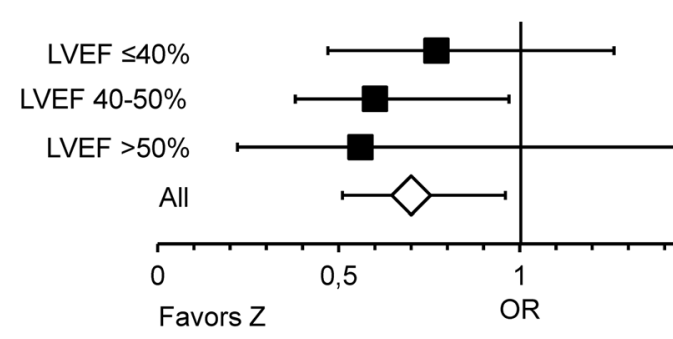

$\begin{array}{lll}\begin{array}{l}\text { Zofenopril } \\ \mathrm{n}(\%)\end{array} & \begin{array}{c}\text { Ramipril } \\ \mathrm{n}(\%)\end{array} & \begin{array}{c}\text { OR } \\ (95 \% \mathrm{Cl})\end{array} \\ 57 / 151(38) & 49 / 111(44) & 0.77(0.47,1.26) \\ 38 / 173(22) & 61 / 192(32) & 0.60(0.38,0.97) \\ 10 / 40(25) & 16 / 43(37) & 0.56(0.22,1.45) \\ 105 / 365(29) & 128 / 351(37) & 0.70(0.51,0.96) \\ 1,5 & & \\ & & \end{array}$

Fig. 4 Cumulative survival without events during 1 year in patients (a) with preserved systolic function (left ventricular ejection fraction $>40 \%)$ and (b) with impaired systolic function (left ventricular ejection fraction $\leq 40 \%$ ) treated with zofenopril (continuous line) or ramipril (dotted line). $\mathbf{c}$ Odds ratio and 95\% confidence interval for 1-year combined occurrence of cardiovascular mortality or hospitalization for cardiovascular causes according to ranges of left ventricular ejection fraction [38]. CI confidence interval, $L V E F$ left ventricular ejection fraction, $O R$ odds ratio, $R$ ramipril, $Z$ zofenopril

(b)

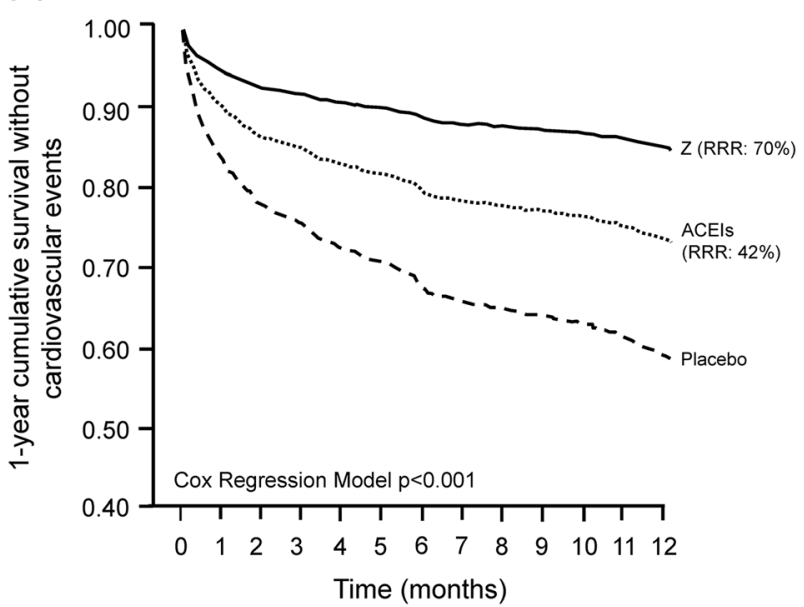

continuous line) in the SMILE program. The relative risk reduction versus placebo is indicated. $p$ values from a Cox regression model [39]. $Z$ zofenopril, $A C E I$ angiotensinconverting enzyme inhibitor, $R R R$ relative risk reduction patients treated with placebo ( $n=951$, dashed lines), other angiotensin-converting enzyme inhibitors (lisinopril or ramipril, $n=871$, dotted lines $)$ and zofenopril $(n=1808$,

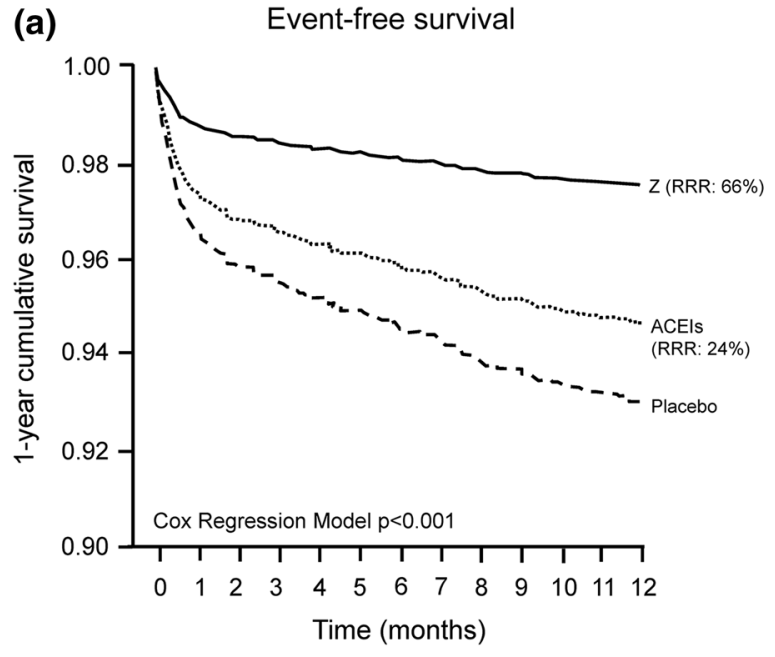

Fig. 5 a Cumulative survival and $\mathbf{b}$ cumulative survival without cardiovascular events during 1-year of follow-up in 


\section{ACKNOWLEDGEMENTS}

Funding. Sponsorship for the SMILE programme and open access fee were funded by Menarini International Operations Luxembourg S.A. No Rapid Service Fee was received by the journal for the publication of this article. The funding source did not influence or commented on planned methods, protocol, data analysis and the paper. All authors had full access to all of the data in this study and take complete responsibility for the integrity of the data and accuracy of the data analysis.

Authorship. All named authors meet the International Committee of Medical Journal Editors (ICMJE) criteria for authorship for this article, take responsibility for the integrity of the work as a whole, and have given their approval for this version to be published.

Disclosures. Conflict of interest of Prof Claudio Borghi: consultancy for Boheringer Ingelheim, Menarini International, Sanofi,

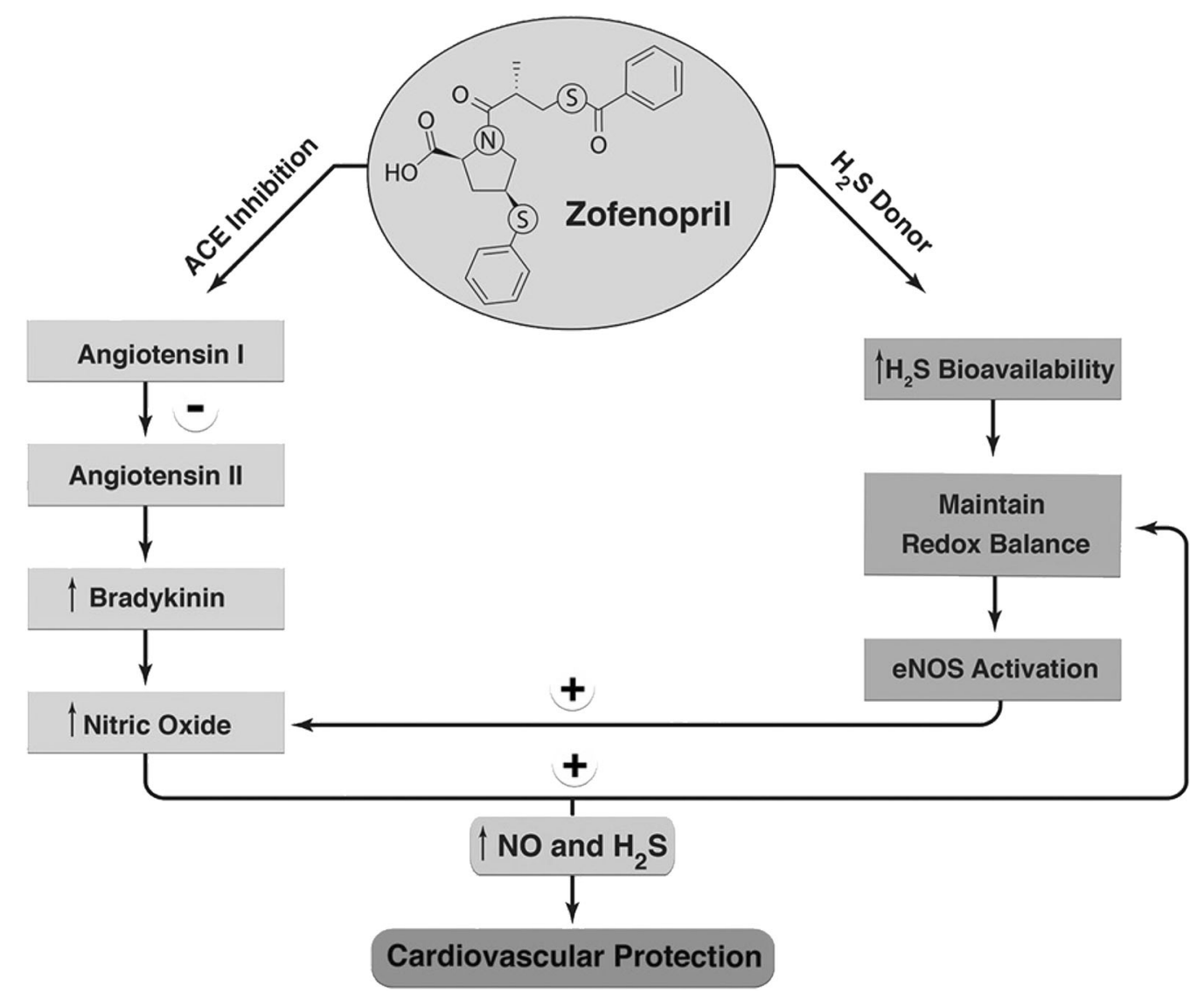

Fig. 6 Effect of zofenopril on hydrogen sulfide and nitric oxide bioavailability. By inhibiting myocardial angiotensinconverting enzyme activity, zofenopril reduces the generation of angiotensin II and increases levels of bradykinin. Bradykinin, through stimulation of endothelial B2 receptors, promotes the release of nitric oxide, prostacyclin, and endothelium-derived hyperpolarizing factor, which in turn leads to cardioprotection. On the other hand, zofenopril, by releasing hydrogen sulfide, enhances tissue antioxidant defense, and promotes activation of endothelial nitric oxide synthase, leading to increased levels of nitric oxide. Therefore, angiotensin-converting enzyme inhibition, hydrogen sulfide, and nitric oxide account for zofenoprilmediated cardioprotective effects [40]. $A C E$ angiotensinconverting enzyme, $B K$ bradykinin, $E D H F$ endotheliumderived hyperpolarizing factor, eNOS endothelial nitric oxide synthase, $H 2 S$ hydrogen sulfide, $N O$ nitric oxide 


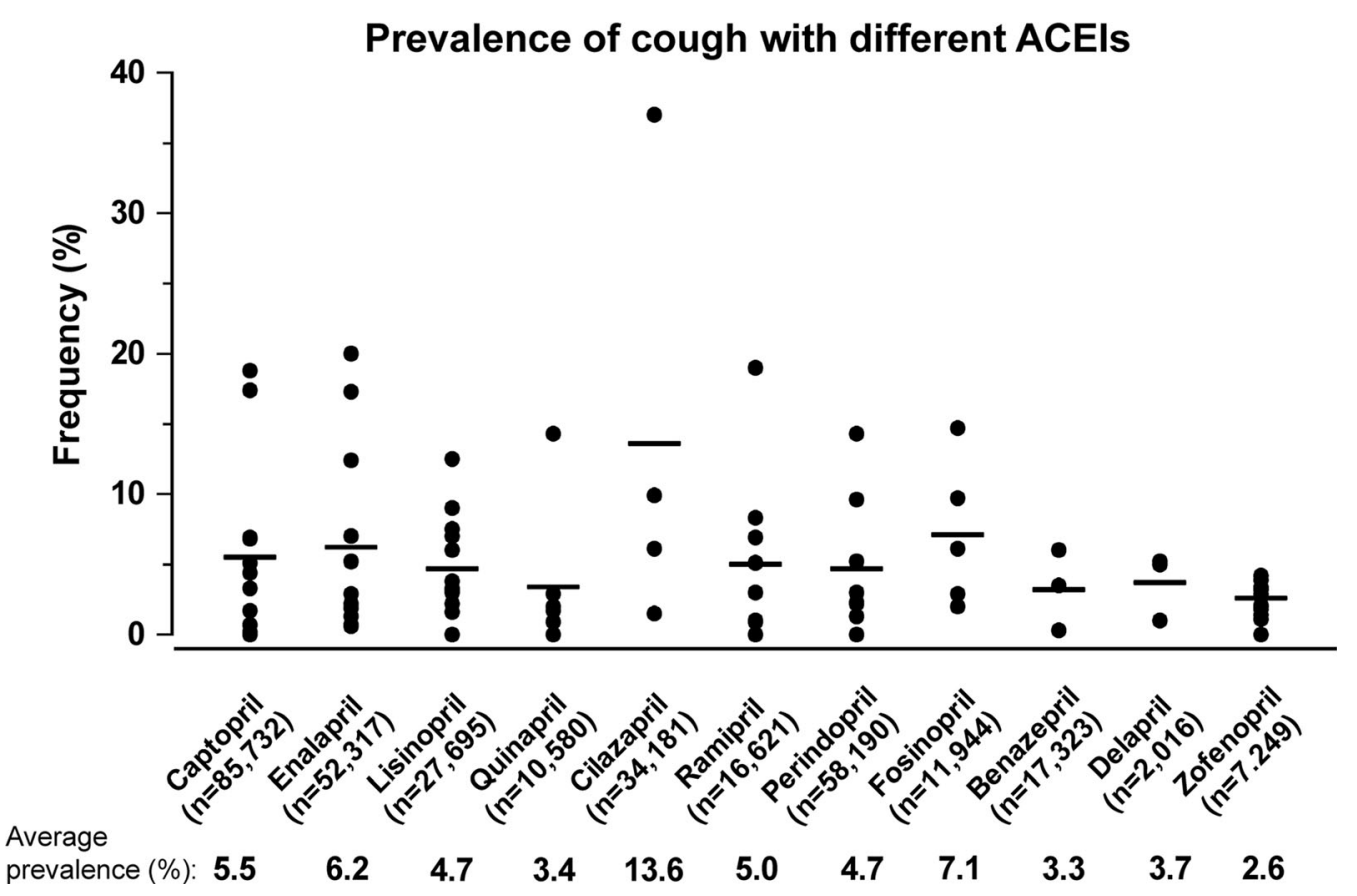

Fig. 7 Prevalence of cough with different ACEIs in double-blind randomized and post-marketing studies and meta-analyses [42]. ACEI angiotensin-converting enzyme inhibitor

Amgen, Takeda, Novartis, Ely Lilly and Servier. Stefano Omboni has nothing to disclose.

Compliance with Ethics Guidelines. This article is based on previously conducted studies and does not contain any studies with human participants or animals performed by any of the authors.

Data Availability. Data sharing is not applicable to this article as no datasets were generated or analyzed during the current study.

Open Access. This article is licensed under a Creative Commons Attribution-NonCommercial 4.0 International License, which permits any non-commercial use, sharing, adaptation, distribution and reproduction in any medium or format, as long as you give appropriate credit to the original author(s) and the source, provide a link to the Creative Commons licence, and indicate if changes were made. The images or other third party material in this article are included in the article's Creative Commons licence, unless indicated otherwise in a credit line to the material. If material is not included in the article's Creative Commons licence and your intended use is not permitted by statutory regulation or exceeds the permitted use, you will need to obtain permission directly from the copyright holder. To view a copy of this licence, visit http://creativecommons.org/licenses/by$\mathrm{nc} / 4.0 /$.

\section{REFERENCES}

1. Kalantarinia K, Okusa MD. The renin-angiotensin system and its blockade in diabetic renal and cardiovascular disease. Curr Diab Rep. 2006;6(1):8-16.

2. Abuissa $\mathrm{H}, \mathrm{O}^{\prime}$ Keefe $\mathrm{J}$ Jr. The role of renin-angiotensin-aldosterone system-based therapy in diabetes prevention and cardiovascular and renal protection. Diabetes Obes Metab. 2008;10(12): 1157-66.

3. Otto CM. Heartbeat: renin-angiotensin system blockade for prevention of cardiovascular disease. Heart. 2017;103:1305-7.

4. Atlas S. The renin-angiotensin aldosterone system: pathophysiological role and pharmacologic inhibition. J Manag Care Pharm. 2007;13:9-20. 
5. Carey RM, Siragy HM. Newly recognized components of the renin-angiotensin system: potential roles in cardiovascular and renal regulation. Endocr Rev. 2003;24:261-7.

6. Gheblawi M, Wang K, Viveiros A, et al. Angiotensin converting enzyme 2: SARS-CoV-2 receptor and regulator of the renin-angiotensin system. Circ Res. 2020;126(10):1456-74.

7. Magellan. Angiotensin modulators: ACE inhibitors and direct renin inhibitors review. Cincinnati: Provider Synergies, LLC; 2012. p. 1-28.

8. Khalil ME, Basher AW, Brown EJ Jr, Alhaddad IA. A remarkable medical story: benefits of angiotensinconverting enzyme inhibitors in cardiac patients. J Am Coll Cardiol. 2001;37(7):1757-64.

9. Ferrario CM, Mullick AE. Renin angiotensin aldosterone inhibition in the treatment of cardiovascular disease. Pharmacol Res. 2017;125(Pt A):57-71.

10. Williams B, Mancia G, Spiering W, et al. Authors/ Task Force Members: 2018 ESC/ESH Guidelines for the management of arterial hypertension: The Task Force for the management of arterial hypertension of the European Society of Cardiology and the European Society of Hypertension: The Task Force for the management of arterial hypertension of the European Society of Cardiology and the European Society of Hypertension. J Hypertens. 2018;36(10): 1953-2041.

11. Gohlke P, Scholkens BA. ACE inhibitors: pharmacology. In: Unger T, Scholkens BA, editors. Angiotensin. Berlin: Springer; 2004. p. 375-413.

12. Davis R, Coukell A, McTavish D. Fosinopril. A review of its pharmacology and clinical efficacy in the management of heart failure. Drugs. 1997;54(1):103-16.

13. Heran BS, Wong MMY, Heran IK, Wright J. Blood pressure lowering efficacy of angiotensin converting enzyme (ACE) inhibitors for primary hypertension. Cochrane Database Syst Rev. 2008;4: CD003823.

14. Kostis JB, Davis BR, Cutler J, et al. Prevention of heart failure by antihypertensive drug treatment in older persons with isolated systolic hypertension. JAMA. 1997;278:212-6.

15. Ponikowski P, Voors AA, Anker SD, et al. ESC Scientific Document Group. 2016 ESC Guidelines for the diagnosis and treatment of acute and chronic heart failure: The Task Force for the diagnosis and treatment of acute and chronic heart failure of the European Society of Cardiology (ESC) Developed with the special contribution of the Heart Failure
Association (HFA) of the ESC. Eur Heart J. 2016;37(27):2129-200.

16. Flather MD, Yusuf S, Køber L, et al. Long-term ACEinhibitor therapy in patients with heart failure or left-ventricular dysfunction: a systematic overview of data from individual patients. Lancet. 2000;355: $1575-81$.

17. Al-Mallah MH, Tleyjeh IM, Abdel-Latif AA, Weaver WD. Angiotensin-converting enzyme inhibitors in coronary artery disease and preserved left ventricular systolic function: a systematic review and meta-analysis of randomized controlled trials. J Am Coll Cardiol. 2006;47(8):1576-83.

18. Knuuti J, Wijns W, Saraste A, et al. ESC Scientific Document Group. 2019 ESC Guidelines for the diagnosis and management of chronic coronary syndromes. Eur Heart J. 2020;41(3):407-77.

19. Pfeffer M. ACE inhibitors in acute myocardial infarction patient selection and timing. Circulation. 1998;97:2192-4.

20. Indications for ACE inhibitors in the early treatment of acute myocardial infarction: systematic overview of individual data from 100,000 patients in randomized trials. ACE Inhibitor Myocardial Infarction Collaborative Group. Circulation. 1998;97(22):2202-12.

21. Hansen ML, Gislason GH, Køber L, et al. Different angiotensin-converting enzyme inhibitors have similar clinical efficacy after myocardial infarction. Br J Clin Pharmacol. 2008;65(2):217-23.

22. Ibanez B, James S, Agewall S, et al. ESC Scientific Document Group. 2017 ESC Guidelines for the management of acute myocardial infarction in patients presenting with ST-segment elevation: The Task Force for the management of acute myocardial infarction in patients presenting with ST-segment elevation of the European Society of Cardiology (ESC). Eur Heart J. 2018;39(2):119-77.

23. Wu HY, Huang JW, Lin HJ, et al. Comparative effectiveness of renin-angiotensin system blockers and other antihypertensive drugs in patients with diabetes: systematic review and bayesian network meta-analysis. Version 2. BMJ. 2013;347:f6008.

24. Chiurchiu C, Remuzzi G, Ruggenenti P. Angiotensin-converting enzyme inhibition and renal protection in nondiabetic patients: the data of the meta-analyses. J Am Soc Nephrol. 2005;16(Suppl 1): S58-63.

25. DeForrest JM, Waldron TL, Krapcho J, et al. Preclinical pharmacology of zofenopril, an inhibitor of angiotensin I converting enzyme. J Cardiovasc Pharmacol. 1989;13:887-94. 
26. Ambrosioni E, Borghi C, Magnani B. Early treatment of acute myocardial infarction with angiotensin-converting enzyme inhibition: safety considerations. SMILE pilot study working party. Am J Cardiol. 1991;68:D101-10.

27. Ambrosioni E, Borghi C, Magnani B. The effect of the angiotensin-converting enzyme inhibitor zofenopril on mortality and morbidity after anterior myocardial infarction. The Survival of Myocardial Infarction Long-Term Evaluation (SMILE) Study Investigators. $\mathrm{N}$ Engl J Med. 1995;332:80-5.

28. Borghi C, Bacchelli S, Degli Esposti D, Bignamini A, Magnani B, Ambrosioni E. Effects of the administration of an angiotensin-converting enzyme inhibitor during the acute phase of myocardial infarction in patients with arterial hypertension. SMILE Study Investigators. Survival of myocardial infarction long-term evaluation. Am J Hypertens. 1999;12:665-72.

29. Borghi C, Bacchelli S, Degli Esposti D, Ambrosioni E, SMILE Study. Effects of the early ACE inhibition in diabetic nonthrombolyzed patients with anterior acute myocardial infarction. Diabetes Care. 2003;26:1862-8.

30. Borghi C, Cicero AF, Bacchelli S, Degli Esposti D, Ambrosioni E, Survival of Myocardial Infarction Long-term Evaluation (SMILE) Study. Serum cholesterol levels on admission and survival in patients with acute myocardial infarction treated with zofenopril: a post hoc analysis of the Survival of Myocardial Infarction Long-term Evaluation trial. Fundam Clin Pharmacol. 2009;23:641-8.

31. Borghi C, Cicero AF, Ambrosioni E. Effects of early treatment with zofenopril in patients with myocardial infarction and metabolic syndrome: the SMILE Study. Vasc Health Risk Manag. 2008;4: 665-71.

32. Borghi C, Bacchelli S, Degli ED, Ambrosioni E. Survival of Myocardial Infarction Long-Term Evaluation Study. Effects of early angiotensin-converting enzyme inhibition in patients with non-STelevation acute anterior myocardial infarction. Am Heart J. 2006;152:470-7.

33. Borghi C, Ambrosioni E. Survival of Myocardial Infarction Long-term Evaluation-2 Working Party. Double-blind comparison between zofenopril and lisinopril in patients with acute myocardial infarction: results of the Survival of Myocardial Infarction Long-term Evaluation-2 (SMILE-2) study. Am Heart J. 2003;145:80-7.

34. Borghi C, Ambrosioni E, Novo S, Vinereanu D, Ambrosio G. SMILE-4 Working Party. Comparison between zofenopril and ramipril in combination with acetylsalicylic acid in patients with left ventricular systolic dysfunction after acute myocardial infarction: results of a randomized, double-blind, parallel-group, multicenter, European study (SMILE-4). Clin Cardiol. 2012;35(7):416-23.

35. Borghi C, Omboni S, Novo S, Vinereanu D, Ambrosio G, Ambrosioni E. Early treatment with zofenopril and ramipril in combination with acetyl salicylic acid in patients with left ventricular systolic dysfunction after acute myocardial infarction: results of a 5-year follow-up of patients of the SMILE-4 Study. J Cardiovasc Pharmacol. 2017;69(5): 298-304.

36. Borghi C, Omboni S, Novo S, Vinereanu D, Ambrosio G, Ambrosioni E, SMILE-4 Working Party. Zofenopril and ramipril in patients with left ventricular systolic dysfunction after acute myocardial infarction: a propensity analysis of the Survival of Myocardial Infarction Long-term Evaluation (SMILE) 4 study. J Renin Angiotensin Aldosterone Syst. 2016;17(4):1470320316656480.

37. Borghi C, Ambrosioni E, Survival of Myocardial Infarction Long-term Evaluation Study Group. Effects of zofenopril on myocardial ischemia in post-myocardial infarction patients with preserved left ventricular function: the Survival of Myocardial Infarction Long-term Evaluation (SMILE)-ISCHEMIA study. Am Heart J. 2007;153:445.e7-14.

38. Borghi C, Omboni S, Cicero AF, et al. Randomised comparison of zofenopril and ramipril plus acetylsalicylic acid in postmyocardial infarction patients with left ventricular systolic dysfunction: a post hoc analysis of the SMILE-4 Study in patients according to levels of left ventricular ejection fraction at entry. Open Heart. 2015;2(1):e000195.

39. Borghi C, Omboni S, Reggiardo G, Bacchelli S, Degli Esposti D, Ambrosioni E. Cardioprotective role of zofenopril in patients with acute myocardial infarction: a pooled individual data analysis of four randomised, double-blind, controlled, prospective studies. Open Heart. 2015;2(1):e000220.

40. Donnarumma E, Ali MJ, Rushing AM, et al. Zofenopril protects against myocardial ischemiareperfusion injury by increasing nitric oxide and hydrogen sulfide bioavailability. J Am Heart Assoc. 2016;5(7):e003531.

41. Cushman DW, Wang FL, Fung WC, Harvey CM, DeForrest JM. Differentiation of angiotensin-converting enzyme (ACE) inhibitors by their selective inhibition of ACE in physiologically important target organs. Am J Hypertens. 1989;2(4):294-306.

42. Omboni S, Borghi C. Zofenopril and incidence of cough: a review of published and unpublished data. Ther Clin Risk Manag. 2011;7:459-71. 